\title{
ENHANCED NETWORK PERFORMANCE AND MOBILITY MANAGEMENT OF IOT MULTI NETWORKS
}

\author{
Dr. M. Durai Pandian, \\ Professor, Department of CSE, \\ Vivekananda College of Engineering, \\ Namakal , India. \\ Email id: svsduraipandian@gmail.com
}

\begin{abstract}
The tremendous progress in the internet of things incorporating multitudes of wireless interfaces have ended up with dense deployment of multi-networks that are associated with the internet of things and supported by wireless communications that are heterogeneous. Effective management of the multi-networks with a proper flow scheduling and a mobility management in order to provide a seamless conveyance are a tedious and a challenging task. The incompatibility of the software defined networks to manage with the densely deployed multi networks has increased the necessity for developing a more suitable flow scheduler and mobility manager for the multi networks of internet of things. So this paper presents the Improved- flow scheduling and the mobility management protocol (IFSMM) to guarantee the network performance that provides a seamless accessing of internet of things. The IFSMM is simulated using the NS-2 to confirm the performance achievement through the proper management of the transmission on the grounds of throughput, latency and the jitter.
\end{abstract}

Keywords: Internet of Things, Multi-Networks, Flow-Schedule, Mobility- Management, Improved Throughput, Improved Jitter, Improved Latency, Software Defined Networks.

\section{INTRODUCTION}

The progressing advancements and the tremendous developments in the in the wireless communication has made the internet of things more popular. Moreover the ubiquitousness of the consumer devices has kindled the interest of more and more users towards the internet of things. This has also increased the applications associated with the internet of things in the rural as well as the urban areas. The real time applications of the internet of things incorporate different networks access and connectivity as they are anticipated to be heterogeneous. The heterogeneous internet of things application connecting using multiple radio-communications such as the Wi-Fi, Bluetooth and the cellular results with the framing multi networks that is spread geographically. The variety found

ISSN: $2582-4104$ 
Journal of trends in Computer Science and Smart technology (TCSST) (2019)

Vol.01/ No. 02 Pages: 95- 105

https://www.irojournals.com/tcsst/

DOI: https://doi.org/10.36548/jtcsst.2019.2.003

in the multi-networks introduces challenges in the optimization and the co-operation in the utilization of resources that are heterogeneous.

The dense deployment of the IOT-multinetworks (IOT-MN) in the urban as well as the rural areas causes a very heavy traffic flow that is changing. So this has become a very prominent area of research, the researcher's attained multiple solutions that improves the configuration and the access of the network devices but found to be unsuitable due to the excess power consumption and the limited power availability in the service networks. The software defined networking [1] that is found to present the possible remedies in managing the flow, updating and storing of huge flow of information's also remains unsuitable and incompatible for the densely deployed heterogeneous multi networks. The figure.1 below shows the overall frame work of the SDN

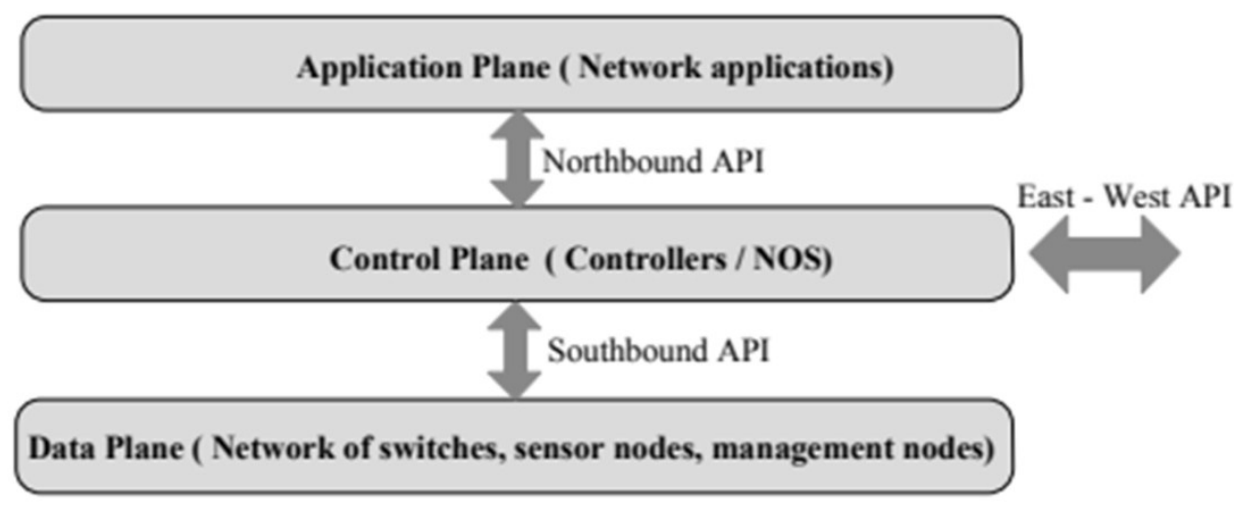

Fig .1 Frame Work of SDN [1]

The prevailing software defined networks implementation appears to be unsuitable for the handling the entailments of the ubiquitous applications of the internet of things due to their heterogeneity.

So in this paper the Improved- flow scheduling and the mobility management protocol (IFSMM) to guarantee the network performance that provides a seamless accessing of internet of things is put forth to manage the heterogeneous-multi network internet of things applications that are geographically wide spread.

ISSN: 2582-4104 
Journal of trends in Computer Science and Smart technology (TCSST) (2019)

Vol.01/ No. 02 Pages: 95- 105

https://www.irojournals.com/tcsst/

DOI: https://doi.org/10.36548/jtcsst.2019.2.003

The remaining of the paper is organized as follows

The section II explains the related works on the prevailing SDN networks and the challenges in SDN in managing the heterogeneous-multi network internet of things applications.

The Section III provides the explanation on the proposed Improved- flow scheduling and the mobility management protocol (IFSMM) to regulate the flow and the mobility of the IOT-MN.

The Section IV presents the result analysis of the proposed IFSMM and its comparison with the prevailing SDN

The Section V is the conclusion followed by the references.

\section{RELATED WORKS}

$\mathrm{Yu}$, et al [2], presents the "survey on the state of the art and future directions for the ultra-dense networks" Ojo, et al [3], presents the "implementation of the network virtualization in the software defined networks" Bera, et al [4], details the entailment of the "software defined networks for the internet of things"

Liu et al [5], elaborates the "Device-to-device communications for enhancing quality of experience in software defined multi-tier LTE-A networks." Hakiri, et al [6], puts forth the "'Leveraging SDN for the 5G networks: trends, prospects and challenges." Uddin et al [7], details the "The SCALE2 multi-network architecture for IoTbased resilient communities."Aloi, et al [8], presents the "details of the multi-networks based on the mobile multitechnology gateway to enable IoT interoperability."

Jonsson,et al [9], presents the "Telecommunications method for multi-network communications" and Vaziri et al [10], provides the "Multi-network exchange system for telephony applications." The "Network slicing based 5G and future mobile networks: mobility, resource management, and challenges." Is proposed by Zhang et al [11], Kobo, et al [12], elaborates the "survey on software-defined wireless sensor networks: Challenges and design requirements."Pandian et al [13] puts forth the "Enhanced Edge Model for Big Data in the Internet of Things Based Applications." Bhalaji et al [14] puts forward the "Delay Diminished Efficient Task Scheduling and Allocation for Heterogeneous Cloud Environment."

ISSN: $2582-4104$ 
Journal of trends in Computer Science and Smart technology (TCSST) (2019)

Vol.01/ No. 02 Pages: 95- 105

https://www.irojournals.com/tcsst/

DOI: https://doi.org/10.36548/jtcsst.2019.2.003

The author S. Smys et al [15] puts forward the "Performance analysis of heuristic clustered (HC) architecture in wireless networks." he also details the recently established "Inventive Systems on Smart Cities." To manage the heterogeneity in the network [16] Raj, Jennifer S et al [17] and [18] proposes the "A new distributed architecture for connectivity analysis in wireless networks." and the "Dynamic Adaptive Topology Control in Highly Mobile Environment" to enhance the connectivity and the control of the network. The table.1 provides the details of the challenges related to the software defined network in supporting the IOT-MN. Bhalaji, et al [21] in his paper elaborates the "Delay Diminished Efficient Task Scheduling and Allocation for Heterogeneous Cloud Environment"

\begin{tabular}{|l|l|}
\hline $\begin{array}{c}\text { S.N } \\
\text { O }\end{array}$ & \multicolumn{1}{|c|}{ Challenges of SDN - IOT -Multi network } \\
\hline 1. & $\begin{array}{l}\text { In capable of Addressing large scale IOT- Multi } \\
\text { networks }\end{array}$ \\
\hline 2. & Lack of scalable control with the consistent management \\
\hline 3. & $\begin{array}{l}\text { The requirement of the controller to be fault tolerant } \\
\text { on failure or traffic congestion. }\end{array}$ \\
\hline 4. & $\begin{array}{l}\text { Heterogeneity in the IOT-MN than the prevailing Wi- } \\
\text { Fi/LTE }\end{array}$ \\
\hline 5. & $\begin{array}{l}\text { Lack of co-ordination and communication due to } \\
\text { redundancy found in IOT-MN }\end{array}$ \\
\hline 6. & $\begin{array}{l}\text { Difference in the requirement of the applications and the } \\
\text { heterogeneity in the capability of the network }\end{array}$ \\
\hline 7. & $\begin{array}{l}\text { Improper Traffic balance between the controller and the } \\
\text { controlled devices. }\end{array}$ \\
\hline
\end{tabular}

Table.1 Challenges of the Software Defined Networks [6], [11], [12]

ISSN: $2582-4104$ 
Journal of trends in Computer Science and Smart technology (TCSST) (2019)

Vol.01/ No. 02 Pages: 95- 105

https://www.irojournals.com/tcsst/

DOI: https://doi.org/10.36548/jtcsst.2019.2.003

\section{PROPOSED IMPROVED- FLOW SCHEDULING AND THE MOBILITY MANAGEMENT PROTOCOL (IFSMM)}

The proposed method to improve the seamless access of the internet of things devices in the distributed software defined network puts forth a protocol to enhance the scheduling and managing the mobility of the IOT-MN. The proposed method initiates with the clustering of the network based on the geographical locations, followed by the selection of the optimal access-points as the consumer devices with the ubiquitousness are related various types of accessing-points such as the Bluetooth, WiMAX, Wi-Fi, cellular etc. and establish local connection using variety of switches in-order to appeal for different for forms of data flow. The large-scale SDN is employed to monitor the complete data flow that takes place under the different clusters and the number of devices added and removed from the clusters and maintains the details in a local controlling device that makes the information's of the number of users and the average utilization of the resources available to all the clusters.

The proposed architecture involves the components such as the controller, switches, access points and the IOT devices. The controller in the control plane monitors the data flow into the network and gathers its description about the network from the IOT-MN and saves it in the network. The proposed protocol is now utilized to cluster the network and schedule the flow by proper selection of the access points within the cluster with minimum delay $\left(\operatorname{Min}_{\text {del }}\right)$, maximum energy $\left(\operatorname{Max}_{\text {ene }}\right)$, minimum mobility $\left(\operatorname{Min}_{\text {mobi }}\right)$ and high throughput $\left(\right.$ high $\left.h_{\text {thpt }}\right)$. The fig.2 below provides flow chart of the proposed process.

ISSN: $2582-4104$ 
Journal of trends in Computer Science and Smart technology (TCSST) (2019)

Vol.01/ No. 02 Pages: 95- 105

https://www.irojournals.com/tcsst/

DOI: https://doi.org/10.36548/jtcsst.2019.2.003

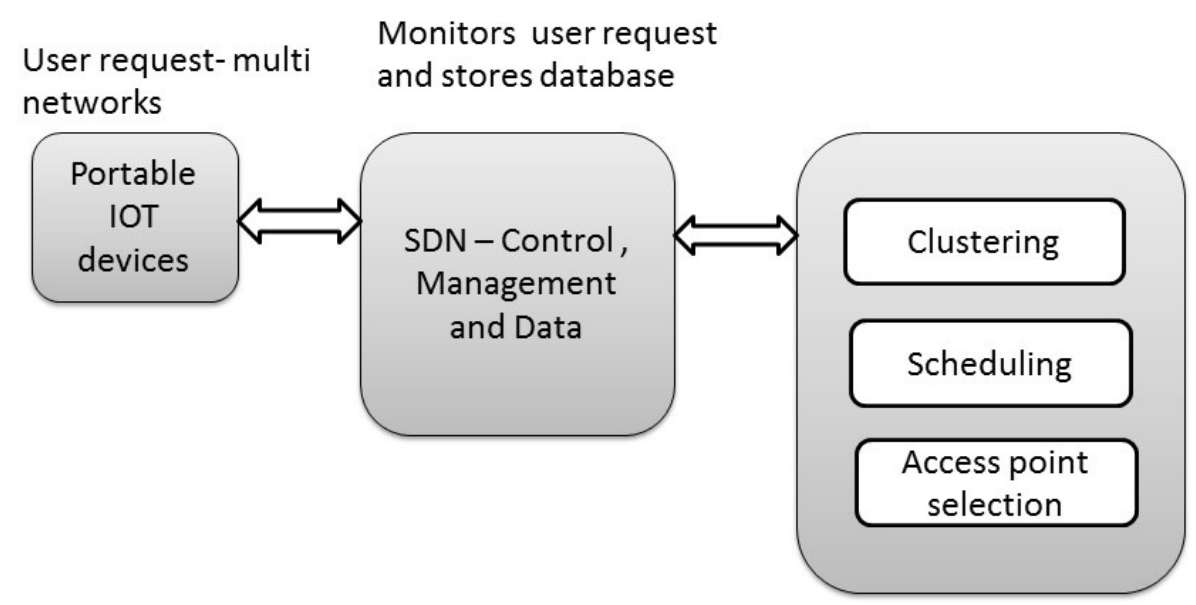

Fig .1 Flow Chart of the Proposed Process

The request entering into the network through the data layer is monitored by the control layer and stored in the data collection center. The proposed method now initiates the clustering of the network using the K-means clustering [19] based on the geographical location and employs a control head to every cluster. Now the heuristic scheduling algorithm based on the PSO (particle swarm optimization) [20] is utilized to determine the befitting access-point with the minimum delay $\left(\operatorname{Min}_{d e l}\right)$, maximum energy $\left(\operatorname{Max}_{e n e}\right)$, minimum mobility $\left(\operatorname{Min}_{m o b i}\right)$ and high throughput $\left(\right.$ high $\left.h_{\text {thpt }}\right)$ from all the clusters. The optimal access points selected are used for the conveying of the information.

The controller that monitors the data flow into the network also keeps note of the entry and exit of the users in the each cluster. This enables cluster to have the details of the average resource utilized and the number of users engaged. This monitoring also enables the user to have a seamless IOT access when the person moves from one cluster to the next. The record maintained by the main controller enables the local controller head of the each cluster to get the previous details associated with the user newly entering into the one cluster from another cluster. The fig. 3 shows the proposed algorithm protocol for managing the flow of data in the IOT-MN

ISSN: $2582-4104$ 
Journal of trends in Computer Science and Smart technology (TCSST) (2019)

Vol.01/ No. 02 Pages: 95- 105

https://www.irojournals.com/tcsst/

DOI: https://doi.org/10.36548/jtcsst.2019.2.003

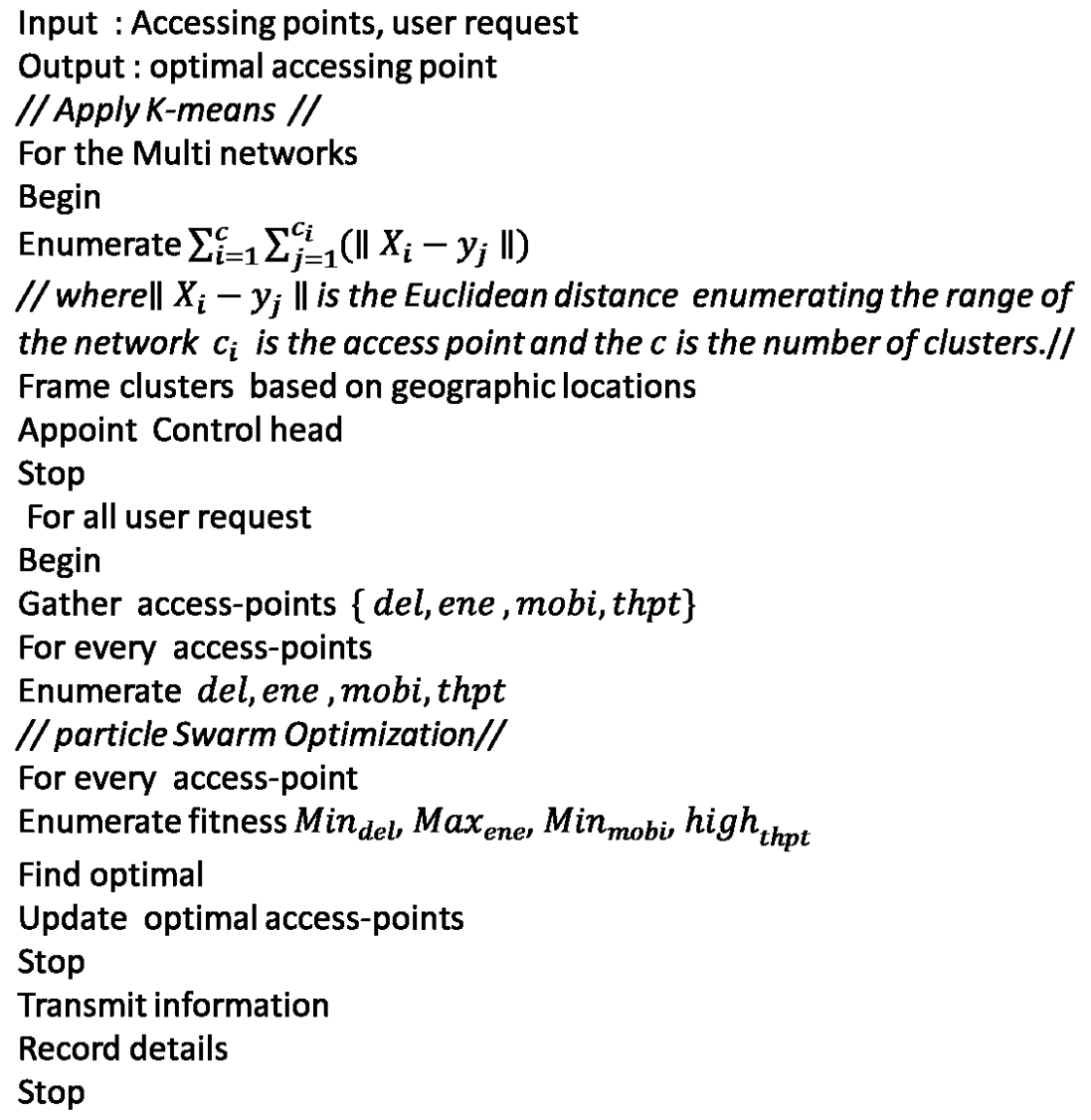

Fig.3 Proposed Protocol

\section{RESULT ANALYSIS}

The proposed method of seamless internet of things access with a proper flow control and the mobility management is evaluated using the network ssimualtor-2 for various numbers of requests ranging 10 to 100 over a simulation time of 2500s under different network configuration such as Wi-Fi, Bluetooth, WiMAX, Zigbee, and cellular to confirm the performance achievement through the proper management of the transmission on the grounds of throughput, latency and the jitter. 
Journal of trends in Computer Science and Smart technology (TCSST) (2019)

Vol.01/ No. 02 Pages: 95- 105

https://www.irojournals.com/tcsst/

DOI: https://doi.org/10.36548/jtcsst.2019.2.003

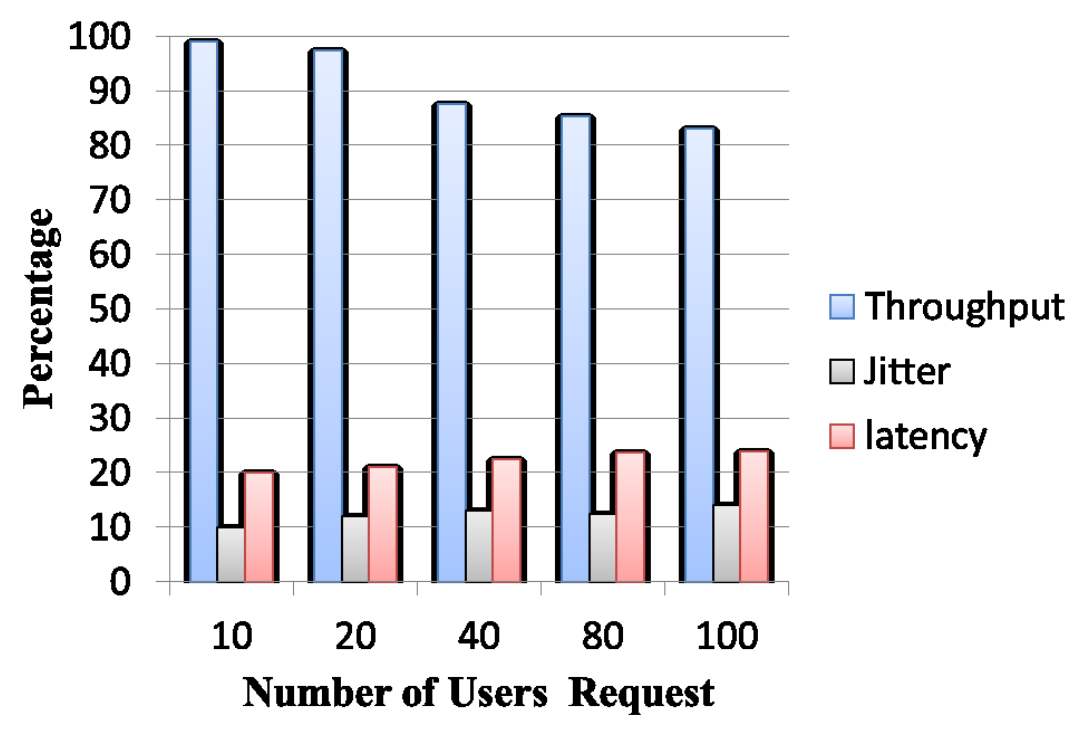

Fig.4 Throughput, Jitter and Latency

The fig. 4 above shows the percentage of the actual values of the throughput, jitter and the latency for the multi networks on using the Improved-Flow Scheduling Mobility Management protocol that enhances the performance by a proper flow scheduling with befitting access point selection.

ISSN: $2582-4104$ 
Journal of trends in Computer Science and Smart technology (TCSST) (2019)

Vol.01/ No. 02 Pages: 95- 105

https://www.irojournals.com/tcsst/

DOI: https://doi.org/10.36548/jtcsst.2019.2.003

\begin{tabular}{|c|c|c|c|c|c|c|c|c|c|}
\hline \multirow[b]{2}{*}{$\begin{array}{c}\text { Number of user } \\
\text { Request }\end{array}$} & \multicolumn{3}{|c|}{ Expected } & \multicolumn{3}{|c|}{ Actual } & \multicolumn{3}{|c|}{ Prevailing-SDN } \\
\hline & $\begin{array}{l}\text { Thro } \\
\text { ughp } \\
\text { ut in } \\
\text { Mbp } \\
\text { s }\end{array}$ & $\begin{array}{l}\text { Jitter } \\
\text { in ms }\end{array}$ & $\begin{array}{l}\text { Late } \\
\text { ncy } \\
\text { in ms }\end{array}$ & $\begin{array}{l}\text { Thro } \\
\text { ughp } \\
\text { ut in } \\
\text { Mbp } \\
\text { s }\end{array}$ & $\begin{array}{l}\text { Jitter } \\
\text { in ms }\end{array}$ & $\begin{array}{l}\text { Late } \\
\text { ncy } \\
\text { in ms }\end{array}$ & $\begin{array}{l}\text { Thro } \\
\text { ughp } \\
\text { ut in } \\
\text { Mbp } \\
\text { S }\end{array}$ & $\begin{array}{l}\text { Jitter } \\
\text { in ms }\end{array}$ & $\begin{array}{l}\text { Late } \\
\text { ncy } \\
\text { in ms }\end{array}$ \\
\hline 10 & 1.4 & $\begin{array}{c}.002 \\
3\end{array}$ & .456 & 1.4 & $\stackrel{.012}{3}$ & .85 & .8 & $\begin{array}{c}.007 \\
8\end{array}$ & .786 \\
\hline 20 & 1.34 & $\begin{array}{c}.002 \\
1\end{array}$ & .487 & 1.4 & $\begin{array}{c}.034 \\
5\end{array}$ & .89 & .97 & $\begin{array}{c}.008 \\
6\end{array}$ & .798 \\
\hline 40 & 1.38 & $\begin{array}{c}.002 \\
5\end{array}$ & .543 & 1.4 & $\begin{array}{c}.032 \\
1\end{array}$ & .90 & .95 & $\underset{9}{.008}$ & .854 \\
\hline 80 & 1.33 & .003 & .563 & 1.4 & .045 & .95 & .986 & $\begin{array}{c}.009 \\
1\end{array}$ & .889 \\
\hline 100 & 1.25 & $\begin{array}{c}.004 \\
5\end{array}$ & .653 & 1.4 & $\begin{array}{c}.057 \\
3\end{array}$ & .98 & .86 & .014 & .854 \\
\hline
\end{tabular}

Table.2 Performance Comparison

The table. 2 shows the performance comparison of the IFSMM with the prevailing SDN for the jitter, throughput and the latency observed for the different number of the user request. The results obtained shows that the proposed IFSMM shows $30 \%$ higher throughput than the expected and the prevailing SDN , $15 \%$ improvement in latency than and $10 \%$ improvement in jitter observed.

\section{CONCLUSION}

This paper presents the seamless accessing of the internet of things in multi-network by proposing an ImprovedFlow Scheduling Mobility Management protocol that enhances the performance by a proper flow scheduling with befitting access point selection using the kmeans and the PSO. The proposed method is simulated using the network simulator-2 to confirm the performance up gradation provided in the SDN for the IOT-MN on the grounds of the throughput, jitter and the latency and it was observed that IFSMM shows $30 \%$ higher throughput than the expected and the prevailing SDN , $15 \%$ improvement in latency and 10\% improvement in jitter. The future work of the paper is to continue developing a load balancing, fault tolerant network for the multi-network-internet of things.

ISSN: $2582-4104$ 
Journal of trends in Computer Science and Smart technology (TCSST) (2019)

Vol.01/ No. 02 Pages: 95- 105

https://www.irojournals.com/tcsst/

DOI: https://doi.org/10.36548/jtcsst.2019.2.003

\section{References}

[1]. Ndiaye, Musa, Gerhard P. Hancke, and Adnan M. Abu-Mahfouz. "Software defined networking for improved wireless sensor network management: A survey." Sensors 17, no. 5 (2017): 1031.

[2]. Yu, Wei, Hansong Xu, Hanlin Zhang, David Griffith, and Nada Golmie. "Ultra-dense networks: Survey of state of the art and future directions." In 2016 25th international conference on computer communication and networks (ICCCN), pp. 1-10. IEEE, 2016.

[3]. Ojo, Mike, Davide Adami, and Stefano Giordano. "A SDN-IoT architecture with NFV implementation." In 2016 IEEE Globecom Workshops (GC Wkshps), pp. 1-6. IEEE, 2016.

[4]. Bera, Samaresh, Sudip Misra, and Athanasios V. Vasilakos. "Software-defined networking for internet of things: A survey." IEEE Internet of Things Journal 4, no. 6 (2017): 1994-2008.

[5]. Liu, Jiajia, Shangwei Zhang, Nei Kato, Hirotaka Ujikawa, and Kenichi Suzuki. "Device-to-device communications for enhancing quality of experience in software defined multi-tier LTE-A networks." IEEE Network 29, no. 4 (2015): 46-52.

[6]. Hakiri, Akram, and Pascal Berthou. "Leveraging SDN for the 5G networks: trends, prospects and challenges." arXiv preprint arXiv:1506.02876 (2015).

[7]. Uddin, Md Yusuf Sarwar, Alexander Nelson, Kyle Benson, Guoxi Wang, Qiuxi Zhu, Qing Han, Nailah Alhassoun et al. "The SCALE2 multi-network architecture for iot-based resilient communities." In 2016 IEEE International Conference on Smart Computing (SMARTCOMP), pp. 1-8. IEEE, 2016.

[8]. Aloi, Gianluca, Giuseppe Caliciuri, Giancarlo Fortino, Raffaele Gravina, Pasquale Pace, Wilma Russo, and Claudio Savaglio. "A mobile multi-technology gateway to enable IoT interoperability." In 2016 IEEE First International Conference on Internet-of-Things Design and Implementation (IoTDI), pp. 259-264. IEEE, 2016.

[9]. Jonsson, Bjorn Erik Rutger. "Telecommunications method for multi-network communications." U.S. Patent 5,915,224, issued June 22, 1999.

[10]. Vaziri, Faramarz, Herbert R. Graefe, Xin Wang, Andrew Pletch, and Jeffrey H. Paige. "Multi-network exchange system for telephony applications." U.S. Patent 7,715,413, issued May 11, 2010.

[11]. Zhang, Haijun, Na Liu, Xiaoli Chu, Keping Long, Abdol-Hamid Aghvami, and Victor CM Leung. "Network slicing based 5G and future mobile networks: mobility, resource management, and challenges." IEEE Communications Magazine 55, no. 8 (2017): 138-145.

ISSN: $2582-4104$ 
Journal of trends in Computer Science and Smart technology (TCSST) (2019)

Vol.01/ No. 02 Pages: 95- 105

https://www.irojournals.com/tcsst/

DOI: https://doi.org/10.36548/jtcsst.2019.2.003

[12]. Kobo, Hlabishi I., Adnan M. Abu-Mahfouz, and Gerhard P. Hancke. "A survey on software-defined wireless sensor networks: Challenges and design requirements." IEEE access 5 (2017): 1872-1899.

[13]. Pandian, A. Pasumpon. "ENHANCED EDGE MODEL FOR BIG DATA IN THE INTERNET OF THINGS BASED APPLICATIONS." Journal of trends in Computer Science and Smart technology (TCSST) 1, no. 01 (2019): 63-73.

[14]. Bhalaji, N. "DELAY DIMINISHED EFFICIENT TASK SCHEDULING AND ALLOCATION FOR HETEROGENEOUS CLOUD ENVIRONMENT." Journal of trends in Computer Science and Smart technology (TCSST) 1, no. 01 (2019): 51-62.

[15]. Ramesh, S., and S. Smys. "Performance analysis of heuristic clustered (HC) architecture in wireless networks." In 2017 International Conference on Inventive Systems and Control (ICISC), pp. 1-4. IEEE, 2017.

[16]. Smys, S., Hui-Ming Wee, and Meng Joo. "Introduction to the Special Section on Inventive Systems and Smart Cities." (2018): 32-33.

[17]. Raj, Jennifer S., and R. Harikumar. "A new distributed architecture for connectivity analysis in wireless networks." Procedia Engineering 30 (2012): 394-401.

[18]. Kuruvila, Diya Ann, and Jennifer S. Raj. "Dynamic Adaptive Topology Control in Highly Mobile Environment."

[19]. Wagstaff, Kiri, Claire Cardie, Seth Rogers, and Stefan Schrödl. "Constrained k-means clustering with background knowledge." In Icml, vol. 1, pp. 577-584. 2001.

[20]. Kennedy, J., and R. Eberhart. "Particle swarm optimization (PSO)." In Proc. IEEE International Conference on Neural Networks, Perth, Australia, pp. 1942-1948. 1995.

[21]. Bhalaji, N. "DELAY DIMINISHED EFFICIENT TASK SCHEDULING AND ALLOCATION FOR HETEROGENEOUS CLOUD ENVIRONMENT." Journal of trends in Computer Science and Smart technology (TCSST) 1, no. 01 (2019): 51-62.

ISSN: 2582-4104 